\title{
GLORIA - THE GLOBAL ROBOTIC TELESCOPES INTELLIGENT ARRAY FOR E-SCIENCE
}

\author{
L. Mankiewicz ${ }^{1}$ on behalf of the GLORIA collaboration
}

\begin{abstract}
GLORIA stands for "GLObal Robotictelescopes Intelligent Array" GLORIA will be the first free and open-access network of robotic telescopes in the world It will be a Web 2.0 environment where users can do research in astronomy by observing with robotic telescopes and/or analyzing data that other users have acquired with GLORIA, or from other free access databases, like the European Virtual Observatory.
\end{abstract}

\section{Introduction}

Many Internet communities have already formed to speedup scientific research, to collaborate in documenting something or as social projects. Research in astronomy can only benefit by attracting many eyes to the sky - to detect something new in the sky requires looking in the right place and the right moment Our robotic telescopes can search the sky but the vast quantities of data produced are far greater than astronomers have time to analyze. Furthermore, even the most advanced and powerful algorithms used in automatic analysis pipelines have limitations that could lead to missing important discoveries. GLORIA will provide a way of putting thousands of eyes and minds on this problem It is intended to be a Web 20 structure with the possibility of doing real experiments The community will not only generate content as in most Web 2.0 but will control telescopes around the world both directly and via scheduled observations The community will take decisions for the network and that will give "intelligence" to GLORIA, while the drudge work (such as drawing up telescope schedules that satisfy various constraints) will be done by dedicated algorithms that are being developed for the purpose.

During the initial period, the GLORIA consortium consists with 13 institutions, operating 17 telescopes which will be integrated into the GLORIA network.

1 Centre for Theoretical Physics of the Polish Academy of Sciences, Al. Lotnikow 32/46, 02-668 Warsaw, Poland 
Some of them, like BOOTES, Pi of the Sky or Mini-MegaTORTORA have observations of optical activity associated with Gamma Ray Bursts as their pricipal scientific program. In the future, we plan to provide userrequired inputs so other users can integrate their telescopes into the network. GLORIA is also collaborating with the "Discover the COSMOS" EU funded project, which aims at innovative ways to involve teachers and students in eScience through existing infrastructures.

The GLORIA project will define free standards, protocols and methodologies for:

1. Controlling robotic telescopes and all related instrumentation such as cameras filterwheels domes etc..

2. Giving web access to the network: access to an arbitrary number of robotic telescopes via a web portal.

3. Conducting on-line experiments: users will be able to design specific web environments to control telescopes and perform observations aimed at studying some specific scientific issues.

4. Conducting off-line experiments: users will be able to design specific web environments for analyzing astronomical meta - data produced by GLORIA and other databases.

We are going to seek the collaboration of amateur astronomers and their telescopes. Some amateur astronomers are very active with excellent instrumentation and observing locations However they are frequently limited in their ability to properly exploit and interpret their data Interaction with professional astronomers and the use of powerful customized analysis tools will greatly improve the quantity, quality and reliability of the data that amateurs can collect with their instruments The GLORIA community will benefit from their telescopes the data they produce, and simply the extra knowledge, experience and ideas their presence will bring.

GLORIA associates with each user through a meritocratic parameter, called "Karma" in Web 2.0 environments This is correlated with the user's activity and their performance of useful work In the context of GLORIA, it is the method whereby access to scarce resources (such as telescope time) is determined. Calculation of karma is done automatically, incorporating, for example, the votes of the community for the work of each user. This method has been successfully proven in many collaborative web sites such as YouTube.

\section{GLORIA as a network}

Technically, GLORIA aims to create a completely robotised and autonomous network of telescopes. For each target, the selection of instrument as well as exposure settings are to be decided automatically, without human intervention, based on information about telescopes and the time slots that each telescope offers for the network. Putting together 17 telescopes in one network offers significant potential 


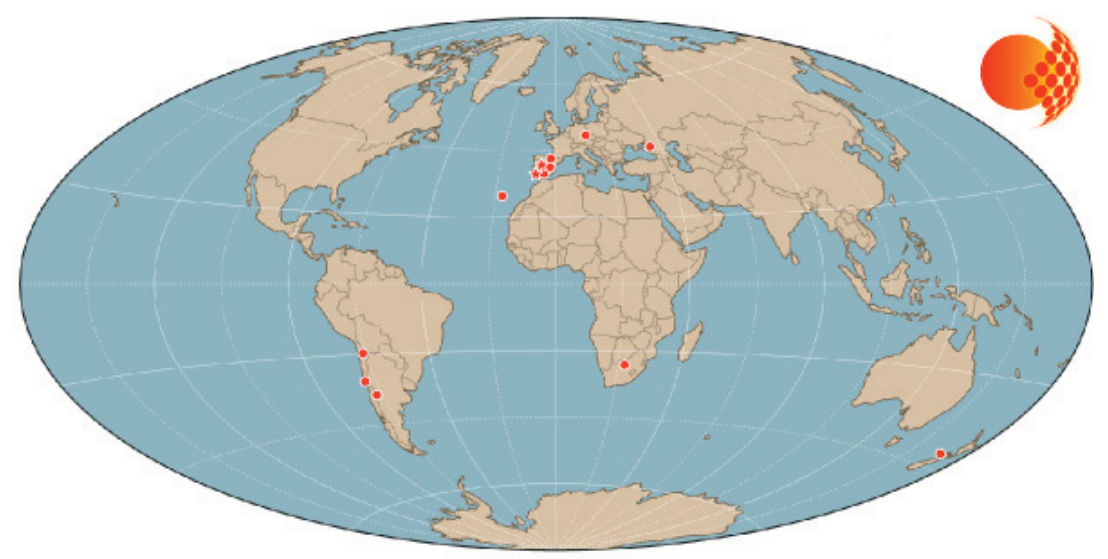

Fig. 1. Geographical location of the initial 17 telescopes of GLORIA. A star symbol means multiple telescopes.

for automatically triggered follow-up observations. Such observations are obviously useful for transients of all kinds, including GRB observations.

\section{Experiments with GLORIA}

GLORIA will provide a web interface that will allow users to perform specific astronomical research projects through "experiments" Users will be guided through the different tasks each project requires. These experiments are of two kinds: those that require a telescope (which we have called "on-line" experiments) and "off-line" experiments, which work on data produced by the GLORIA network or derived from other databases, such as the European Virtual Observatory. Moreover, GLORIA will design a methodology documentation and software components to allow users to design new experiments Advanced users will not only be able broadto design new experiments, but also to integrate them into the network, by following the open methodology, and make them usable by all.

During the lifetime of the GLORIA project there will be demonstrators for at least one online and one off-line experiment At an early stage of the project the partners will decide on the exact astronomical topics to start investigating, including, for example: Exoplanets, Supernovae Gravitational lenses, PHA (Potentially Hazardous Asteroids) Space debris, NEO (Near Earth Objects) etc..

\section{Broadcasted astronomical events}

To advertise the project and test P2P technologies, GLORIA had set the goal to broadcast live 5 astronomical events around the world. Three such broadcasts, the Venus Transit June $6^{\text {th }} 2012$, Northern Lights from Greenland August 
$24^{\text {th }}-28^{\text {th }} 2012$, and Solar Eclipse November $13^{\text {th }} 2012$ have been successfuly performed. Thanks to innovative P2P technology the GLORIA network delivered images and video streams taken in Japan, Australia and Norway live to more than 100 thousand viewers. In addition, some commercial TV stations took the GLORIA stream and broadcast it on their channel. Dedicated pedagogical materials were prepared and distributed prior to each event and off-line experiments were created using data and images collected during the event. For further information visit gloria-project.eu

GLObal Robotic telescopes Intelligent Array for e-Science (GLORIA) is a project funded by the European Union Seventh Framework Programme (FP7/2007-2012) under grant number 283783. I am grateful to A.J. Castro-Tirado, L. Hanlon, L. Nicastro and F.M. Sanchez for their remarks concerning the manuscript. 